\title{
Extended one-step block method for solving stiff initial value problem
}

\begin{abstract}
An extended 2-point one-step block method formula with order four is formulated for solving stiff initial value problem. The method is similar to Runge-Kutta method which has a selfstarting formula. The approximation solutions at two points will be computed simultaneously by integrating the coefficients over the closest point in the block. The accuracy and the stability properties of the method will be investigated. The numerical results show the efficiency of the proposed method in terms of accuracy when solving stiff initial value problems.
\end{abstract}

Keyword: Block method; Constant step size; Initial value problems; One-step method; Stiff 